\title{
Subaortic aneurysm after arterial switch operation for transposition type double outlet right ventricle
}

\author{
Hisayuki Hongu, MD, PhD, ${ }^{a}$ Masaaki Yamagishi, MD, PhD, ${ }^{a}$ Yoshinobu Maeda, MD, ${ }^{a}$ and \\ Hitoshi Yaku, MD, PhD, ${ }^{\mathrm{b}}$ Kyoto, Japan
}

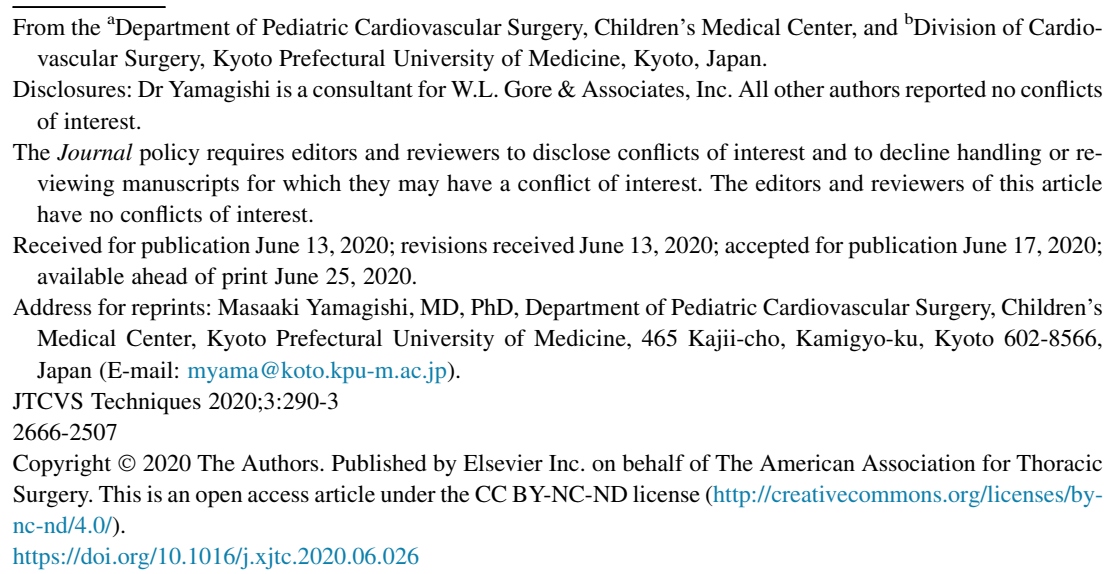
Video clip is available online.

Left ventricular outflow tract (LVOT) aneurysm is rare and has been reported in association with postcardiac surgery trauma or infective endocarditis. ${ }^{1-3}$ We have reported a case of asymptomatic LVOT aneurysm of the ventricular infundibular fold (VIF) that had been present between the aortomitral annulus, which exists only in the presence of a double outlet right ventricle (DORV). The aneurysm had been detected after complete repair of transposition of the great arteries (TGA) and DORV. To the best of our knowledge, the present report is the first of LVOT aneurysm in the VIF between the aortomitral annulus.

\section{CASE REPORT}

The patient's family provided written informed consent. A 6-year-old boy had been referred to our institution for the management of an LVOT aneurysm. The postnatal diagnosis had been TGA-type DORV and patent ductus arteriosus. Previously, he had undergone ligation of the ductal ligament at the age of 15 days and an arterial switch operation and complete closure of a ventricular septal defect (VSD) at the age of 2 months. He had had an uneventful recovery, had been asymptomatic at follow-up examinations, and required no medications.

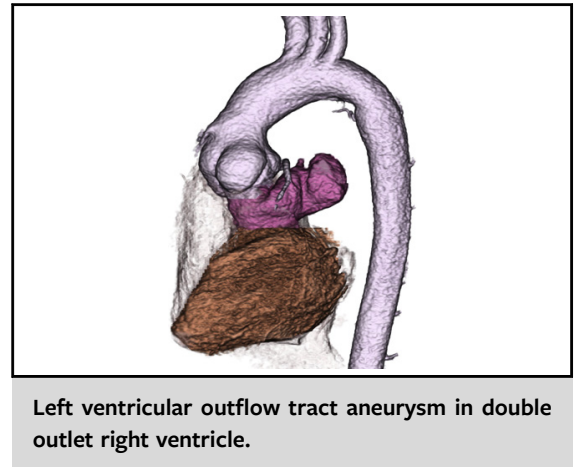

\begin{abstract}
CENTRAL MESSAGE
Case report of an idiopathic subaortic true aneurysm in the ventricular infundibular fold that was detected after an arterial switch operation for transposition type double outlet right ventricle.
\end{abstract}

See Commentary on page 294.

At 6 years of follow-up, cardiac catheterization and cardiac computed tomography that had been performed as routine investigations before he was to enter elementary school revealed a hitherto unidentified LVOT aneurysm. The saccular mass measured $22 \times 20 \mathrm{~mm}$ and was located in the space surrounding the ascending aorta, left pulmonary artery, and left atrium. The left circumflex branch ran superiorly across the mass. The aneurysm orifice was located in the VIF between the mitral and aortic annulus (Video 1). On cardiac catheterization, the aneurysm showed paradoxical motion (Figure 1).

Through a median sternotomy and cardiopulmonary bypass, transverse aortotomy was performed in the subaortic area after achieving cardiac arrest (Figure 2, A). From the aortotomy, the aneurysm orifice was found in the VIF beneath the aortic annulus. The orifice was located at the contralateral side of the interventricular septum and far from the VSD patch. No signs of endocarditis or surgical trauma were seen around the orifice, and no thrombus was identified in the aneurysm (Figure 2,B). Aortic or mitral 


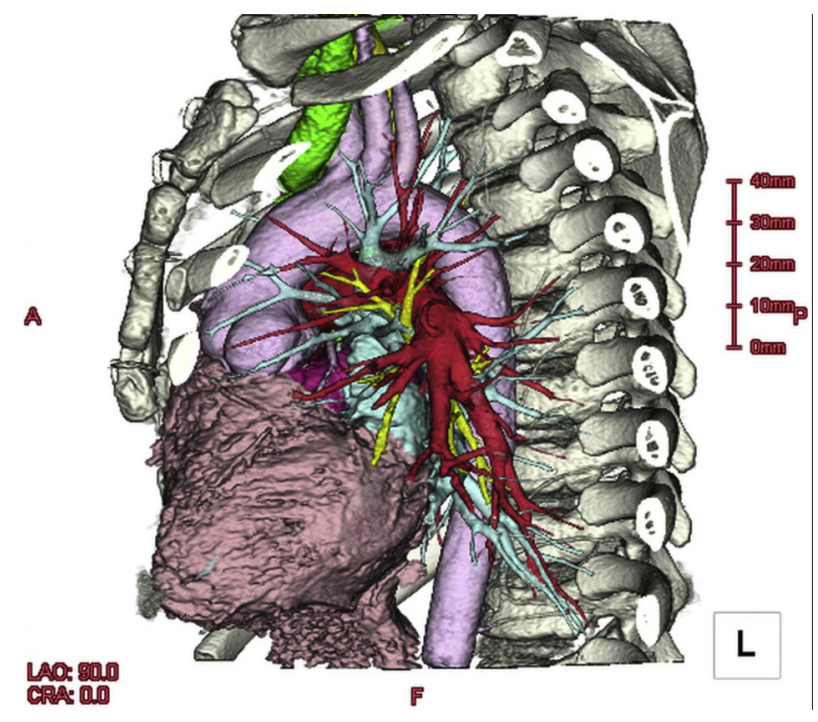

VIDEO 1. Coronal and sagittal slices of preoperative cardiac 3dimensional computed tomography scan. Video available at: https:// www.jtcvs.org/article/S2666-2507(20)30304-7/fulltext.

annular deformities were absent. The aneurysm body was resected from an external approach. The aortomitral space was enlarged, and the aneurysm orifice was closed using an expanded polytetrafluoroethylene patch with interrupted polypropylene sutures (Figure 2, $C$; Video 2). No obvious LVOT obstruction was identified. At completion of the repair, no evidence was found of any residual aneurysm, and the aortic and mitral valves were found to be competent (Video 3). The boy had an uneventful postoperative recovery and was discharged on postoperative day 12 . No evidence was found of aneurysm recurrence at the follow-up examination after 1 year. On histopathologic examination, the resected aneurysm wall was confirmed as a true aneurysm owing to the presence of muscle fibers identified using a desmin stain (Figure 2, D).

\section{DISCUSSION}

LVOT aneurysm is a rare complication of aortic valve replacement, endocarditis, or chest trauma surgery. ${ }^{1-3}$ It has been reported as a pseudoaneurysm that is usually found in the mitral-aortic intervalvular fibrosa. ${ }^{1-4}$ Our patient had undergone an arterial switch operation with coronary transfer as an infant; however, the subaortic LVOT, including the mitral-side ventriculo-infundibular fold, had not been corrected during that operation. VSD closure was performed via the tricuspid valve. The surgical procedures that could result in iatrogenic injury of the subaortic area, such as VSD enlargement and/or resection of the infundibular septum, had not been performed

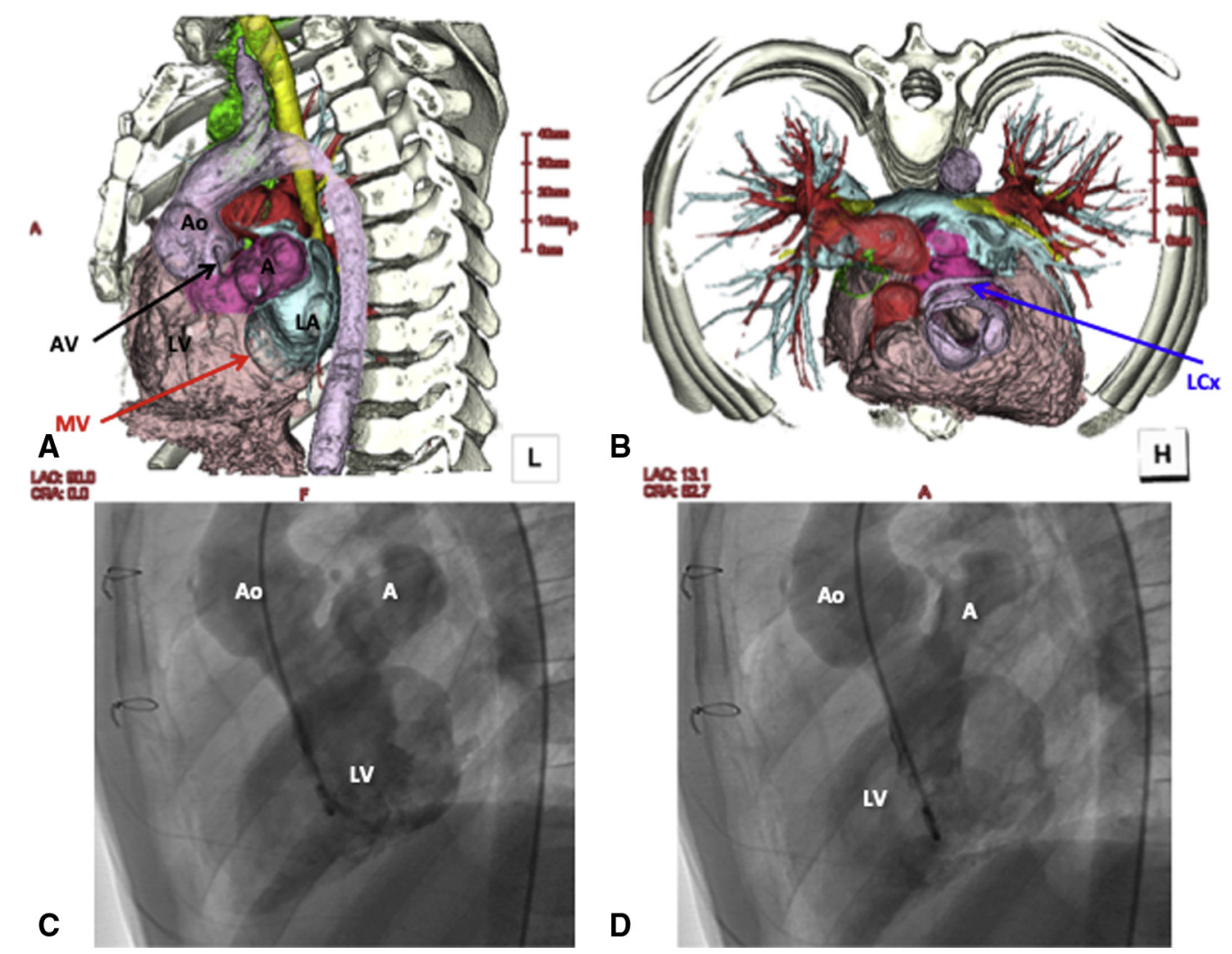

FIGURE 1. Preoperative examination. A and B, Cardiac computed tomography scan showing aortic valve (black arrow), mitral valve (red arrow), and left circumflex branch (blue arrow). C, Cardiac catheterization, systolic phase. D, Cardiac catheterization, diastolic phase. The aneurysm demonstrated paradoxical motion. $A$, Aneurysm; $A V$, aortic valve; $M V$, mitral valve; $L C x$, left circumflex branch; $A o$, ascending aorta; $L V$, left ventricle. 

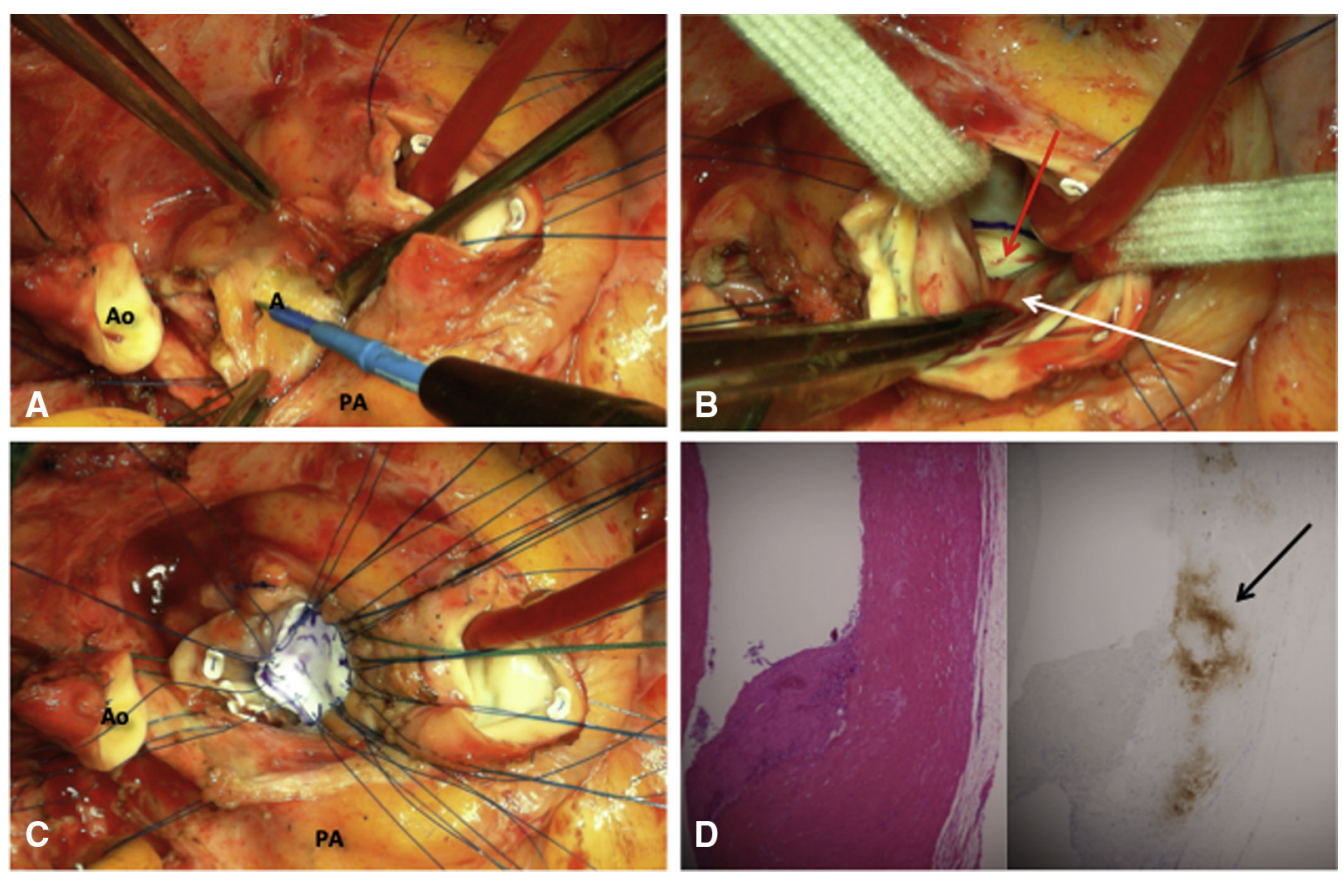

FIGURE 2. Operative and postoperative examination findings. A, Appearance of the aneurysm after division of the aorta (Ao). B, Transaortic valve view showing the orifice of the aneurysm (red arrow) and ventricular infundibular fold between the aortic and mitral annulus (white arrow). C, The aneurysm orifice was closed using an expanded polytetrafluoroethylene patch. D, Pathological diagnosis. Slides of hematoxylin and eosin stain (left) and desmin stain (right). Black arrow indicates the muscle fiber. A, Aneurysm; $P A$, pulmonary artery.

during the previous operation. In addition, the diagnosis had been confirmed as DORV. This implies the presence of muscle tissue in the area and suggests that the connection between the aortic and mitral annulus might be stronger than that in the case of dextro-TGA.

At his previous operation, mobilization of coronary artery had been minimal, and the side small branch had not been sacrificed. In addition, he had no ischemic symptoms and no changes on the electrocardiogram during follow-up. These findings ruled out an ischemic ventricular aneurysm. An untreated aneurysm is at a risk of rupture into the

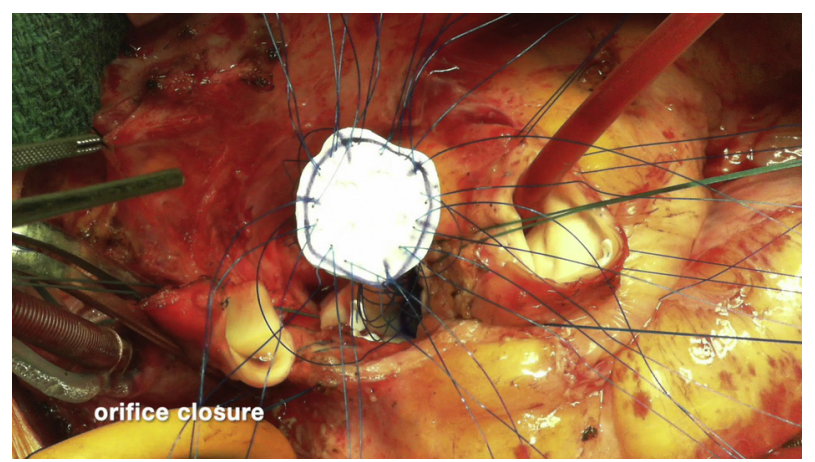

VIDEO 2. Aneurysm resection and complete patch closure of the aneurysmal orifice. Video available at: https://www.jtcvs.org/article/S26662507(20)30304-7/fulltext. pericardium and can result in cardiac tamponade or compression of the surrounding structures, such as the left coronary artery, left atrium, and/or pulmonary artery. ${ }^{5}$ Hence, early surgical repair is desirable once the diagnosis has been confirmed.

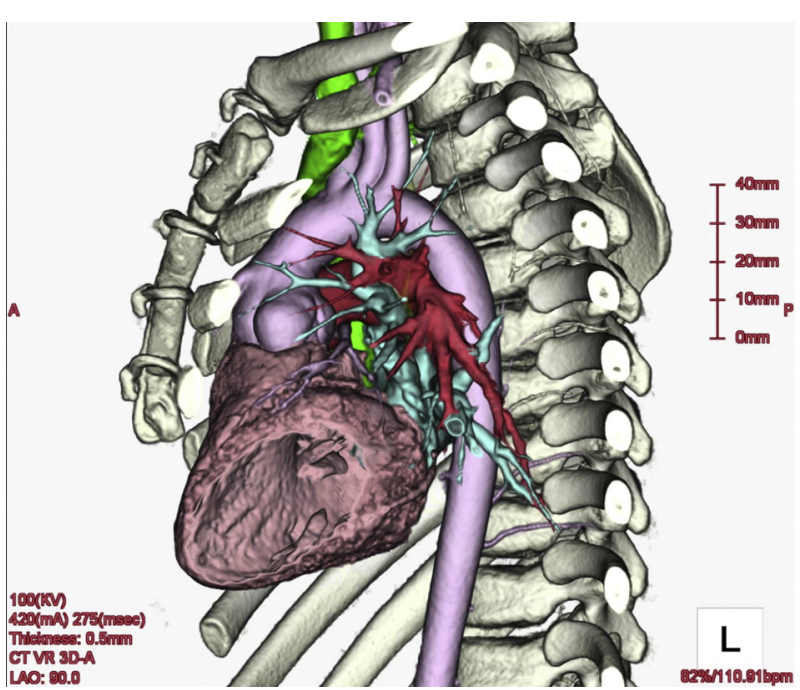

VIDEO 3. Coronal and sagittal slices of postoperative cardiac 3dimensional computed tomography scan. Video available at: https:// www.jtcvs.org/article/S2666-2507(20)30304-7/fulltext. 


\section{References}

1. Espinosa-Caliani JS, Montijano A, Melero JM, Montiel A. Pseudoaneurysm in the mitral-aortic intervalvular fibrosa: a cause of mitral regurgitation. Eur J Cardiothorac Surg. 2000;17:757-9.

2. Fazlinezhad A, Fatehi H, Tabaee S, Alavi M, Hoseini L, Yousefzadeh H. Pseudoaneurysm of mitro-aortic intervalvular fibrosa during the course of mitral valve endocarditis with aorto-left ventricle outflow tract fistula. J Saudi Heart Assoc. 2012; 24:201-4.
3. Bizzarri F, Braconi L, Rossi A, Sorbara C, Stefano PL. Left ventricular outflow tract false aneurysm late after aortic valve replacement. Heart Surg Forum. 2005;8:E136-9.

4. He D, Sinha P, Olivieri L, Jonas RA. Congenital aneurysm of the aortomitral intervalvular fibrosa. Ann Thorac Surg. 2015;99:314-6.

5. Parashara DK, Jacobs LE, Kotler MN, Yazdanfar S, Spielman SR, Janzer SF, et al Angina caused by systolic compression of the left coronary artery as a result of pseudoaneurysm of the mitral-aortic intervalvular fibrosa. Am Heart J. 1995;129:417-21. 